\title{
The Reliability Evaluation of Electromagnetic Valve of EMUs Based on Two-Parameter Exponential Distribution
}

\author{
Jianwei Yang ${ }^{*}, 1,2$, Chunqing Zhao ${ }^{1,2}, \mathrm{Xi} \mathrm{Li}^{3}$ and Fumin Wang ${ }^{4}$ \\ ${ }^{I}$ School of Machine-electricity and Automobile Engineering, Beijing University of Civil Engineering and Architecture, \\ Beijing, 100044, China \\ ${ }^{2}$ Beijing Engineering Research Center of Monitoring for Construction Safety, Beijing University of Civil Engineering \\ and Architecture, Beijing, 100044, China \\ ${ }^{3}$ Subway Operation Technology Centre, Mass Transit Railway Operation Corporation LTD, Beijing, 102208, China \\ ${ }^{4}$ School of Mechanical Engineering, Taiyuan University of Science and Technology, Taiyuan, Shanxi, 030024, China
}

\begin{abstract}
In order to solve reliability evaluation of life of electromagnetic valve of EMUs, this paper evaluated the life of electromagnetic valve under small sample size based on zero-failure data. Firstly, prior distribution of the failure probability was taken into consideration and then the posteriori distribution was obtained by using the Bayes method so that the Bayes estimation could be received under the square loss. Finally, according to the $p_{i}$ received, the reliability parameters of two-parameter exponential distribution were estimated based on weighted least square method. In addition, this paper applied the reliability theory to the reliability life evaluation of electromagnetic valve of EMUs which shows that this method can solve the reliability assessment problem providing certain theoretical basis for the reliability of electromagnetic valve.
\end{abstract}

Keywords: EMUs electromagnetic Valve, Bayes method, Two-parameter exponential distribution, Weighted least square method.

\section{INTRODUCTION}

With the speed increasing by more than $200 \mathrm{~km} / \mathrm{h}$ in operation of EMUs for large area, China Railway has entered the high-speed development stage which is one of the main transportation means in our country. The safety of the system and high-efficiency operation should be ensured when the vehicle is running which are important safeguards of vehicle transportation safety. Prior to estimating the reliability, breakdown should be avoided to guarantee safe and normal operation of the system.

Although the EMUs component has the advantages of high reliability and long life, there can be serious consequences once a failure occurs. So it is necessary to evaluate its reliability life so as to effectively avoid accidents to a certain extent. In reference [1], Zhu Li introduced EMUs reliability index and conducts statistical calculation for breakdown of CRH2 traction system to analyze the reliability; Zhu Dexin used incomplete hybrid Beta distribution as the prior distribution of the failure probability, and solved reliability mathematical model of high-speed train bearing under small sample combining with multi-Bayes evaluation method as shown in reference [2]. In addition, the method of decreasing and increasing function has been put forward by Han Ming to structure prior distribution which provides

*Address correspondence to this author at the School of Machine-electricity and Automobile Engineering, Beijing University of Civil Engineering Architecture, Beijing, 100044, P.R. China Tel: +86 1068322515 ;

E-mail: yangjianwei@bucea.edu.cn a basis for prior distribution [3]; meanwhile, Han Ming makes modified maximum likelihood function and least square estimate for zero-failure data under two-parameter exponential distributions to get the reliability estimation [4]; in reference [5], Zhao Haibing selected $\left(1-p_{2}\right)^{2}$ and $\left(1-p_{2}\right)^{s}$ as the core of $p_{2}$ for the evaluation of the reliability by making use of exponential distribution with no memory; for bearing life which obeys Weibull distribution, Zhu Dexin summarized the relationship of $p_{\mathrm{i}}$ and made an estimation under small sample size to carry out reliability life assessment based on Bayes [6].

In this paper, Bayes method was applied to the reliability evaluation of EMUs electromagnetic valve on the basis of previous studies. It reasonably analyzed the relationship between failure probabilities and considered prior distribution of the failure probability, and then combined priori information and test information to receive posterior distribution so that Bayes estimation of $p_{\mathrm{i}}$ can be obtained under the quadratic loss function. Thus, the reliability parameters can be estimated on the basis of weighted least square method in order to achieve the purpose of the reliability analysis of electromagnetic valve.

\section{THE RELIABILITY MODEL OF EMUS ELECTROMAGNETIC VALVE}

This paper established the reliability model of EMUs electromagnetic valve based on the two-parameter exponential distribution where reliability of the model can be evaluated. 


\subsection{EMUs Electromagnetic Valve}

Rail transport has become an important component of the transportation system in China as a mode of transportation in the long distance with convenience and large capacity. The braking system is one of the important aspects which influences EMUs safety operation, therefore, its life reliability is extremely important.

Electromagnetic valve is automated and is the basic component which belongs to the actuator. It can match different circuits to achieve the desired control. Electromagnetic valve is composed of exhaust valve department and solenoid valve department, which includes two types: ON and OFF, and it makes the movable core move to open or close the exhaust valve by the excitation and demagnetization of solenoid valve coil.

EMUs electromagnetic valve includes emergency braking magnetic valve, EP value and antispin solenoid valve, etc. Sometimes electromagnetic valve indicates some fault, such as: it does not work after getting powered, it does not shut down, internal and external leakage occurs and so on. According to the related statistics, failure rate of the electromagnetic valve in EMUs is higher, and the whole system can be damaged if the electromagnetic valve is not reliable, so it is necessary to evaluate its reliability life.

\subsection{Mathematical Model of System Reliability}

In the reliability theory, one-parameter exponential distribution is widely used, especially for electronic products. Two-parameter exponential distribution is used to represent the fatigue life of the metal products including vehicles and hydraulic equipment [7].

Its life is assumed to obey two-parameter exponential distribution and the distribution function is given as follows [4]:

$F(\mathrm{t})=1-\exp \left(-\frac{t-\mu}{\sigma}\right), \quad t \geq \mu$

where, $F(\mathrm{t})$ is the failure probability, $\mathrm{t}$ is the life of electromagnetic valve which is random variable, $\mu$ and $\sigma$ are parameters of two-parameter exponential distribution.

The density function and reliability function are as follows:

$$
\mathrm{f}(\mathrm{t})=\frac{1}{\sigma} \exp \left(-\frac{t-\mu}{\sigma}\right), \quad t \geq \mu
$$

$$
R(\mathrm{t})=1-F(\mathrm{t})=\exp \left(-\frac{t-\mu}{\sigma}\right), \quad t \geq \mu
$$

The failure rate function is as follows:

$$
\lambda(\mathrm{t})=\frac{\mathrm{f}(t)}{R(t)}=\frac{1}{\sigma}
$$

Under the condition of limited funds and resources, this paper adopted time truncation censoring test to assume EMUs component with long design cycle, expensive cost and high reliability. Time truncation censoring test refers to setting a time in advance, and the life test is conducted for a number of products and stops once the deadline is reached. In time truncation censoring test, the test data received when the failure does not occur is called zero-failure data for products with high reliability and long life. Components reliability index can be estimated using information offered by zero-failure data $[8,9]$.

It assumes the reliability model: time truncation censoring test for $\mathrm{k}$ times is carried out; truncation time meets $\mathrm{t}_{1}<\mathrm{t}_{2}<\cdots<\mathrm{t}_{k}$; the number of test samples in the $\mathrm{i}$-th test is $\mathrm{n}_{i}$ which is zero-failure data. The data can be obtained as follows:

$\left(\mathrm{t}_{i}, \mathrm{~s}_{i}\right) \quad i=1,2, \cdots, k$

where, $\mathrm{s}_{i}=\mathrm{n}_{i}+\mathrm{n}_{i+1}+\cdots+\mathrm{n}_{k}$, and $\mathrm{s}_{i}$ shows the number of samples which have not been malfunctioned at time $t_{i}$.

\section{THE METHOD OF BAYES RELIABILITY EVALUATION}

Compared with the classic methods, the Bayes method makes full use of priori information, especially for expensive and small sample products; it can effectively shorten the test cycle, greatly reducing the test cost and improving the test efficiency, having an extensive application prospect. In addition, how to select a priori distribution is the key to Bayes method which can be seen from Bayes formula. And now the failure probability and the parameter of twoparameter exponential distribution are estimated $[10,11]$.

\subsection{The Estimation of the Failure Probability $p_{i}$}

This paper assumed that failure probability at time $t_{i}$ obeys the binomial distribution, and the likelihood function is as follows:

$$
L\left(\mathrm{r}_{i} \mid p_{i}\right)=C_{s_{i}}^{r_{i}} p_{i}^{r_{i}}\left(1-p_{i}\right)^{s_{i}-r_{i}}
$$

where, $C_{s}^{r_{i}}$ means the combination where there is $r_{i}$ sample which is disabled among $s_{i}$ samples.

And it can be obtained as follows under zero-failure data:

$$
L\left(0 \mid p_{i}\right)=\left(1-p_{i}\right)^{s_{i}}
$$

\subsubsection{The Property of the Failure Probability}

On the basis of concavity and convexity of distribution function, Zhang Zhihua in the reference [12] obtained the relationship of failure probability, and then Bayes estimation of failure probability and population parameters was calculated in the case of the exponential distribution and normal distribution. Using the method of decreasing function, ore of prior distributions of $\mathrm{p}_{\mathrm{i}}$ for two-parameter exponential distribution was chosen [4] on the basis of which this research was carried out. The distribution function of two-parameter exponential distribution is convex function of $\mathrm{t}[4,12]$, so $\mathrm{p}_{0}=0$ can be received when $\mathrm{i}=1, \mathrm{p}_{\mathrm{i}-1}<p_{\mathrm{i}}$ when $\mathrm{t}_{\mathrm{i}-1}<t_{\mathrm{i}}$, and the following can be obtained: 
$\frac{\mathrm{p}_{\mathrm{i}-1}}{\mathrm{t}_{\mathrm{i}-1}}>\frac{\mathrm{p}_{\mathrm{i}}}{\mathrm{t}_{\mathrm{i}}}, \quad \mathrm{i}=2,3, \cdots, m$

Then

$\mathrm{p}_{\mathrm{i}-1}<\mathrm{p}_{\mathrm{i}}<\frac{\mathrm{p}_{\mathrm{i}-1} \mathrm{t}_{\mathrm{i}}}{\mathrm{t}_{\mathrm{i}-1}}, \quad \mathrm{i}=2,3, \cdots, m$

In this paper $c_{i}=\frac{p_{i-1} t_{i}}{t_{i-1}}$ is assumed at the same time, $p_{i-1}$ is replaced by the estimation $\mathrm{p} \hat{\mathrm{i}}-1$. Then the property of failure probability can be obtained as follows:

pi $-1<\mathrm{p}_{\mathrm{i}}<\mathrm{c}_{i}, \quad \mathrm{i}=2,3, \cdots, m$

\subsubsection{The Bayes Estimation of $\mathrm{p}_{1}$}

Through reference [12] and [5], it is obvious that the difference between $p_{i}$ and $\hat{p} \hat{i}$ is minor when $s_{i}$ is major. So $\hat{p}_{1}=\frac{0.5}{\mathrm{~s}_{1}+1}$ is selected as the estimation of $\mathrm{p}_{1}$ in this paper which is reasonable.

\subsubsection{The Bayes Estimation of $\mathrm{p}_{\mathrm{i}}$}

The method of decreasing function has been adopted [12] and $\left(1-p_{2}\right)^{2}$ is selected as the core of prior distribution of failure probability $p_{2}$ on the basis of reference [4] in this paper where $f\left(\hat{\mathrm{p}}_{1}, 1\right)$ is regarded as the prior distribution of $\mathrm{p}_{2}$ in the interval $\left(\hat{p}_{1}, c_{2}\right)$ as follows:

$f\left(p_{2} \mid \mathbf{c}_{2}\right)=f\left(\hat{p}_{1}, c_{2}\right)=\frac{3\left(1-p_{2}\right)^{2}}{\left(1-p_{1}\right)^{3}-\left(1-c_{2}\right)^{3}}$

Besides hyper-parameter, ${ }^{c_{2}}$ obeys uniform distribution from $a_{2}$ to $b_{2}$, and the density function is $f\left(\mathrm{c}_{2}\right)=\frac{1}{a_{2}-b_{2}}\left(a_{2}<\mathrm{c}_{2}<b_{2}\right)$, where, $\mathrm{a}_{2}=\hat{p_{1}}, \mathrm{~b}_{2}=\frac{2 \hat{p_{1}} t_{2}}{t_{1}}$ and $\hat{p}_{1}<\mathrm{p}_{2}$ [4]. Then multilayer prior distribution function of $\mathrm{p}_{2}$ is obtained as follows:

$\mathrm{f}\left(p_{2}\right)=\int_{0}^{1} f\left(p_{2} \mid \mathrm{c}_{2}\right) d c_{2}$

$=\left\{\begin{array}{lc}A\left[g\left(b_{2}\right)-g\left(p_{2}\right)\right]\left(1-p_{2}\right)^{2} & \hat{\mathrm{p}}_{1}<p_{2}<b_{2} \\ 0 & \text { otherwise }\end{array}\right.$

where, $\mathrm{A}=\frac{3}{\mathrm{~b}_{2}-\mathrm{a}_{2}}, \mathrm{~g}(\mathrm{x})=\int_{0}^{x} \frac{d x}{1-(1-x)^{3}}$

In accordance with the Bayes theory, the posteriori distribution of failure probability $p_{2}$ is obtained as follows:

$$
\begin{aligned}
& \pi\left(p_{2}\right)=\frac{\mathrm{f}\left(p_{2}\right) L\left(0 \mid \mathrm{p}_{2}\right)}{\int_{\hat{p_{1}}}^{1} \mathrm{f}\left(p_{2}\right) L\left(0 \mid \mathrm{p}_{2}\right) d p_{2}} \\
& = \begin{cases}{\left[\mathrm{q}\left(a_{2}, b_{2}, s_{2}\right)\right]^{-1}\left[g\left(b_{2}\right)-g\left(p_{2}\right)\right]\left(1-p_{2}\right)^{s_{2}+2}} & \hat{p_{1}<p_{2}<\mathrm{b}_{2}} \\
0 & \text { otherwise }\end{cases}
\end{aligned}
$$

where, $\mathrm{q}\left(a_{2}, b_{2}, s_{2}\right)=\int_{a_{2}}^{b_{2}}\left[g\left(b_{2}\left(-\mathrm{g}\left(a_{2}\right)\right]\left(1-p_{2}\right)^{s_{2}+2} d p_{2}\right.\right.$,

$g(x)=\int_{0}^{x} \frac{d x}{1-(1-x)^{3}}$

Combined with the likelihood function and posterior distribution of the failure probability, multi-Bayes estimation of failure probability $p_{2}$ is given under the quadratic loss function as follows:

$$
\begin{aligned}
& \hat{p_{2}}=\int_{0}^{1} p_{2} \pi\left(p_{2}\right) d p_{2}=\left[\mathrm{q}\left(a_{2}, b_{2}, s_{2}\right)\right]^{-1} \int_{p_{1}}^{b_{2}}\left[g\left(b_{2}\right)-g\left(a_{2}\right)\right] p_{2}\left(1-p_{2}\right)^{s_{2}+2} d p_{2} \\
& =\left[\mathrm{q}\left(a_{2}, b_{2}, s_{2}\right)\right]^{-1}\left[\mathrm{q}\left(a_{2}, b_{2}, s_{2}\right)-\mathrm{q}\left(a_{2}, b_{2}, s_{2}+1\right)\right] \\
& =1-\frac{\mathrm{q}\left(a_{2}, b_{2}, s_{2}+1\right)}{\mathrm{q}\left(a_{2}, b_{2}, s_{2}\right)}
\end{aligned}
$$

Similarly, the hyper-parameter selects uniform distribution from $a_{i}$ to $b_{i}$, where, $\mathrm{a}_{\mathrm{i}}=\hat{p}_{i-1}, \mathrm{~b}_{\mathrm{i}}=\frac{2 \hat{p}_{i-1} t_{i}}{t_{i-1}}$, $\hat{p}_{i-1}<\mathrm{p}_{\mathrm{i}}$, and $f\left(\hat{p}_{i-1}, 1\right)$ is written for prior distribution of $\mathrm{p}_{\mathrm{i}}$ in the interval $\left(\hat{p}_{i-1}, b_{i}\right)$ as follows:

$f\left(p_{\mathrm{i}} \mid \mathrm{c}_{i}\right)=f\left(p_{i-1}, c_{i}\right)=\frac{3\left(1-p_{i}\right)^{2}}{\left(1-p_{i-1}\right)^{3}-\left(1-c_{i}\right)^{3}}$

The multilayer prior distribution function is obtained as follows:

$\mathrm{f}\left(p_{i}\right)=\int_{0}^{1} f\left(p_{i} \mid \mathbf{c}_{i}\right) d c_{i}= \begin{cases}A\left[g\left(b_{i}\right)-g\left(p_{i}\right)\right]\left(1-p_{i}\right)^{2} & \hat{p_{i-1}<p_{i}<b_{i}} \\ 0 & \text { otherwise }\end{cases}$

where, $\mathrm{A}=\frac{3}{\mathrm{~b}_{i}-\mathrm{a}_{i}}, \mathrm{~g}(t)=\int_{0}^{t} \frac{d t}{1-(1-t)^{3}}$

Then the posteriori distribution of $\mathrm{p}_{\mathrm{i}}$ is achieved:

$$
\begin{aligned}
& \pi\left(p_{i}\right)=\frac{\mathrm{f}\left(p_{i}\right) L\left(0 \mid \mathrm{p}_{i}\right)}{\int_{p_{i}}^{1} \mathrm{f}\left(p_{i}\right) L\left(0 \mid \mathrm{p}_{i}\right) d p_{i}} \\
& =\left\{\begin{array}{lc}
{\left[\mathrm{q}\left(a_{i}, b_{i}, s_{i}\right)\right]^{-1}\left[g\left(b_{i}\right)-g\left(p_{i}\right)\right]\left(1-p_{i}\right)^{s_{i}+2}} & \hat{p_{i-1}<p_{i}<b_{i}} \\
0 & \text { otherwise }
\end{array}\right.
\end{aligned}
$$

Under the quadratic loss function, multi-Bayes estimation of the failure probability is as follows:

$$
\begin{aligned}
& \hat{p}_{\mathrm{i}}=\int_{0}^{1} p_{i} \pi\left(p_{i}\right) d p_{i}=\left[\mathrm{q}\left(a_{i}, b_{i}, s_{i}\right)\right]^{-1} \int_{p_{i-1}}^{b_{i}}\left[g\left(b_{i}\right)-g\left(a_{i}\right)\right] p_{i}\left(1-p_{i}\right)^{s_{i}+2} d p_{i} \\
& =\left[\mathrm{q}\left(a_{i}, b_{i}, s_{i}\right)\right]^{-1}\left[\mathrm{q}\left(a_{i}, b_{i}, s_{i}\right)-\mathrm{q}\left(a_{i}, b_{i}, s_{i}+1\right)\right] \\
& =1-\frac{\mathrm{q}\left(a_{i}, b_{i}, s_{i}+1\right)}{\mathrm{q}\left(a_{i}, b_{i}, s_{i}\right)}
\end{aligned}
$$

where, $\mathrm{q}\left(a_{i}, b_{i}, s_{i}\right)=\int_{a_{i}}^{b_{i}}\left[g\left(b_{i}\right)-\mathrm{g}\left(a_{i}\right)\right]\left(1-p_{i}\right)^{s_{i}+2} d p_{i}$ 


\subsection{Weighted Least Square Method of Two-Parameter Exponential Distribution}

The common methods of point estimation include moment estimation, order statistics, least square method, etc. Based on zero-failure data, this paper, after the estimation and obtaining $\mathrm{p}_{\mathrm{i}}$ according to the Bayes method, estimates the parameters of two-parameter exponential distribution using weighted least square method $[7,13]$.

According to $F(t)=1-\exp \left(-\frac{t_{i}-\mu}{\sigma}\right), \ln (1-\mathrm{F}(\mathrm{t}))=-\left(\frac{1}{\sigma} \mathrm{t}_{\mathrm{i}}-\frac{\mu}{\sigma}\right)$ can be obtained. And it is supposed that $\alpha=\frac{\mu}{\sigma}, \beta=-\frac{1}{\sigma}$, $\mathrm{x}_{i}=t_{i}$ and $y_{i}=\ln \left(1-\hat{\mathrm{p}}_{i}\right)$, then $\mathrm{p}_{\mathrm{i}}$ is replaced by $\hat{\mathrm{p}}_{\mathrm{i}}$ which introduces error. So now weighted least square method is used to solve the parameters $\alpha$ and $\beta$, besides it is supposed as follows:

$Q=\sum_{i=1}^{k} \omega_{i}\left[y_{i}-\ln (1-F(t))\right]^{2}=\sum_{i=1}^{k} \omega_{i}\left[y_{i}-\alpha-\beta x_{i}\right]^{2}$

The estimation received in reliability test is related to sample number and testing time. So this paper chooses $\omega_{i}=n_{i} t_{i} / \sum_{i=1}^{k} n_{i} t_{i} \quad(i=1,2 \cdots k)$ as weight coefficient, and then:

$$
\left\{\begin{array}{l}
\frac{\partial Q}{\partial \alpha}=-2 \sum_{i=1}^{k} \omega_{i}\left(y_{i}-\alpha-\beta x_{i}\right)=0 \\
\frac{\partial Q}{\partial \beta}=-2 \sum_{\mathrm{i}=1}^{k} \omega_{i} x_{i}\left(y_{i}-\alpha-\beta x_{i}\right)=0
\end{array}\right.
$$

It is obtained as follows:

$$
\left\{\begin{array}{l}
\hat{\alpha}=\frac{A D-B C}{A^{2}-C E} \\
\hat{\beta}=\frac{A B-D E}{A^{2}-C E}
\end{array}\right.
$$

where, $\quad \mathrm{A}=\sum_{\mathrm{i}=1}^{\mathrm{k}} \omega_{\mathrm{i}} \mathrm{x}_{i} \quad, \quad \mathrm{~B}=\sum_{\mathrm{i}=1}^{\mathrm{k}} \omega_{\mathrm{i}} y_{i}, \quad C=\sum_{\mathrm{i}=1}^{\mathrm{k}} \omega_{\mathrm{i}} \mathrm{x}_{i}{ }^{2}$, $\mathrm{D}=\sum_{\mathrm{i}=1}^{\mathrm{k}} \omega_{\mathrm{i}} \mathrm{x}_{i} \mathrm{y}_{i}, \mathrm{E}=\sum_{\mathrm{i}=1}^{\mathrm{k}} \omega_{\mathrm{i}}$

On the above basis, the estimations of $\mu, \sigma$ are as follows:

$$
\left\{\begin{array}{l}
\hat{\mu}=\frac{A D-B C}{D E-A B} \\
\hat{\sigma}=\frac{A^{2}-C E}{D E-A B}
\end{array}\right.
$$

The reliability and failure rate at any time can be obtained through bringing the calculated value of $\hat{\mu}, \hat{\sigma}$ into Eq. (3) and (4) as follows:

$$
\begin{aligned}
& R(\mathrm{t})=\exp \left(-\frac{t-\hat{\mu}}{\hat{\sigma}}\right) \\
& \lambda(\mathrm{t})=\frac{\mathrm{f}(t)}{R(t)}=\frac{1}{\hat{\sigma}}
\end{aligned}
$$

\section{RELIABILITY ANALYSIS OF EMU $_{S}$ ELECTROMAGNETIC VALVE}

Electromagnetic valves belong to products with high reliability, so this paper adopted zero-failure data in order to avoid a large number of testing expenses. It assumed that the life of electromagnetic valve obeys two-parameter exponential distribution, and now aiming at the reliability life problem of electromagnetic valve, it randomly selected $\mathrm{n}=20$ electromagnetic valves to conduct 8 time truncation censoring tests without zero-failure data in the test results $[14,15]$. The specific zero-failure data is shown in Table $\mathbf{1}$ for the time unit in seconds.

Based on zero-failure data of Fig. (1) and Eq. (13) and (17), the failure probability at each truncated moment can be obtained as shown in Fig. (2). And $\mathrm{a}_{1}=1, b_{1}=\frac{1}{s_{1}+1}$ are selected when $\mathrm{i}=1$.

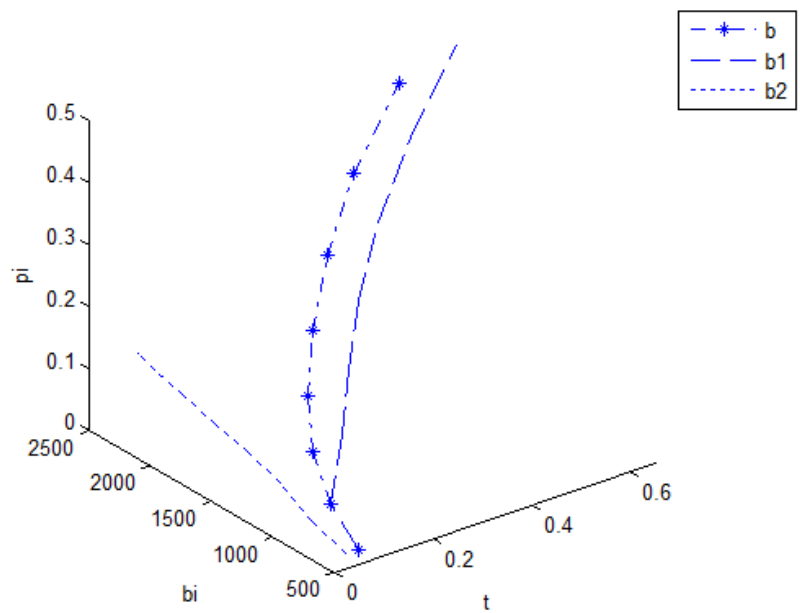

Fig. (1). The comparison of 3D stereogram of failure probability in the three cases.

According to reference [13], this paper chose two other kinds of methods to select hyper-parameter $b$ to compare with the method adopted in this research. It assumed

$$
\begin{aligned}
& \mathrm{b}_{11}=\frac{0.5}{s_{1}+1}, \mathrm{~b}_{12}=\hat{p}_{1} \frac{t_{2}}{t_{1}}, \\
& \mathrm{~b}_{1 i}=\hat{p}_{i-1} \frac{\left(t_{i}+t_{i-1}\right) / 2}{t_{i-2}}, i=3, \cdots, k,
\end{aligned}
$$

and then the estimation of failure probability $\mathrm{p}_{1 i}$ at each truncated moment was obtained as shown in Table 3.

Similarly, it assumed $\mathrm{b}_{21}=\frac{1}{s_{1}+1}, \quad \mathrm{~b}_{22}=2 \hat{p_{1}} \frac{t_{2}}{t_{1}}$, $\mathrm{b}_{2 i}=\hat{p}_{i-1} \frac{\left(t_{i}+t_{i-1}\right)}{t_{i-2}}, i=3, \cdots, k$, and the estimation of 
Table 1. The zero-failure data of electromagnetic valve.

\begin{tabular}{|c|c|c|c|c|c|c|c|}
\hline $\mathbf{i}$ & $\mathbf{1}$ & $\mathbf{2}$ & $\mathbf{3}$ & $\mathbf{4}$ & $\mathbf{5}$ & $\mathbf{6}$ & $\mathbf{7}$ \\
\hline \hline $\mathrm{t}_{i}$ & 500 & 850 & 1200 & 1500 & 1800 & 2100 & 2350 \\
\hline $\mathrm{n}_{i}$ & 2 & 2 & 2 & 2 & 3 & 3 & 3 \\
\hline $\mathrm{s}_{i}$ & 20 & 18 & 16 & 14 & 12 & 9 & 6 \\
\hline
\end{tabular}

Table 2. The estimation of failure probability $p_{i}$ at each truncated moment.

\begin{tabular}{|c|c|c|c|c|c|c|c|c|}
\hline $\mathbf{i}$ & 1 & 2 & 3 & 4 & 5 & 6 & 7 & 8 \\
\hline $\mathrm{a}_{i}$ & 0 & 0.0238 & 0.0468 & 0.0780 & 0.1170 & 0.1647 & 0.2236 & 0.2965 \\
\hline $\mathrm{b}_{i}$ & 0.0476 & 0.0809 & 0.1321 & 0.1950 & 0.2808 & 0.3844 & 0.5005 & 0.6308 \\
\hline $\mathrm{p}_{i}$ & 0.0238 & 0.0468 & 0.0780 & 0.1170 & 0.1647 & 0.2236 & 0.2965 & 0.3909 \\
\hline
\end{tabular}

Table 3. The estimation of failure probability $\mathrm{p}_{1 i}$ at each truncated moment.

\begin{tabular}{|c|c|c|c|c|c|c|c|c|}
\hline $\mathbf{i}$ & $\mathbf{1}$ & $\mathbf{2}$ & $\mathbf{3}$ & $\mathbf{4}$ & $\mathbf{5}$ & $\mathbf{6}$ & $\mathbf{7}$ & $\mathbf{8}$ \\
\hline \hline $\mathrm{a}_{1 i}$ & 0 & 0.0238 & 0.0468 & 0.0780 & 0.1170 & 0.1647 & 0.2236 & 0.2965 \\
\hline $\mathrm{b}_{1 i}$ & 0.0238 & 0.0405 & 0.0648 & 0.0739 & 0.0813 & 0.0904 & 0.0983 & 0.1023 \\
\hline $\mathrm{p}_{1 i}$ & 0.0238 & 0.0316 & 0.0465 & 0.0591 & 0.0695 & 0.0795 & 0.0886 & 0.0954 \\
\hline
\end{tabular}

Table 4. The failure probability estimation $\mathrm{p}_{2 i}$ at each truncated moment.

\begin{tabular}{|c|c|c|c|c|c|c|c|c|}
\hline $\mathbf{i}$ & $\mathbf{1}$ & $\mathbf{2}$ & $\mathbf{3}$ & $\mathbf{4}$ & $\mathbf{5}$ & $\mathbf{6}$ & $\mathbf{7}$ & $\mathbf{8}$ \\
\hline \hline $\mathrm{a}_{2 i}$ & 0 & 0.0238 & 0.0468 & 0.0780 & 0.1170 & 0.1647 & 0.2236 & 0.2965 \\
\hline $\mathrm{b}_{2 i}$ & 0.0476 & 0.0810 & 0.1912 & 0.2802 & 0.3726 & 0.4880 & 0.6158 & 0.7469 \\
\hline $\mathrm{p}_{2 i}$ & 0.0238 & 0.0468 & 0.0882 & 0.1355 & 0.1877 & 0.2491 & 0.3234 & 0.419 \\
\hline
\end{tabular}

failure probability $\mathrm{p}_{2 i}$ at each truncated moment was obtained as shown in Table 4.

So the comparison of 3D stereograms between hyperparameter $b_{i}$ at each truncated moment and failure probability in the three cases supposed was obtained as shown in Fig. (1), in which the curves of $b, b_{1}, b_{2}$ respectively correspond to the values of Tables 2-4. The failure probability received according to the first method to select hyper-parameters is more smooth and continuous than another two, which shows that the selection is more reasonable. Meanwhile, the relationship between failure probability and time is shown in Fig. (2), and the relationship between the failure probability and the hyper-parameters is shown in Fig. (2) in which they basically have a linear correlation. In addition, the estimations of two parameters of two-parameter exponential distribution, reliability and failure rate can be obtained as follows:

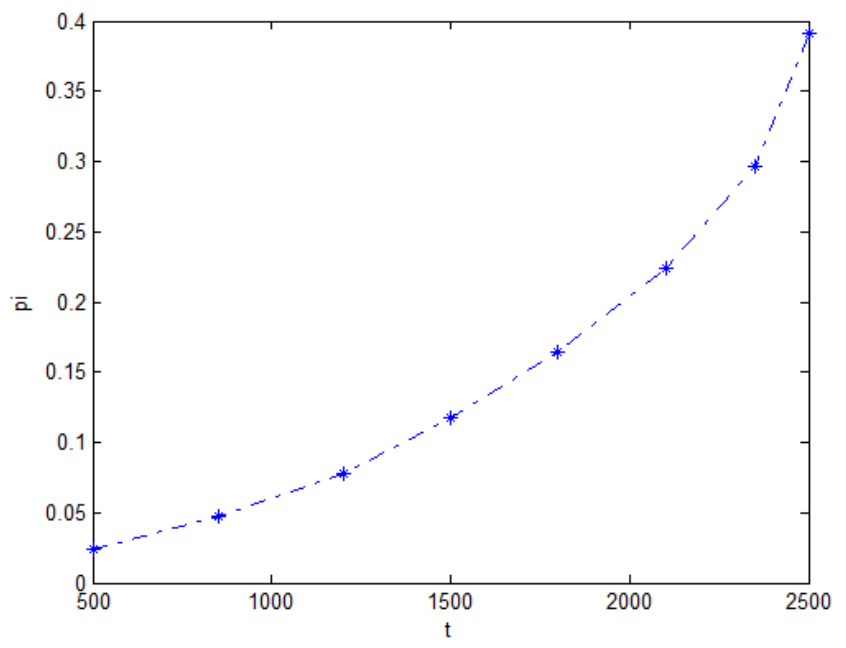

Fig. (2). The relationship between failure probability and time. 


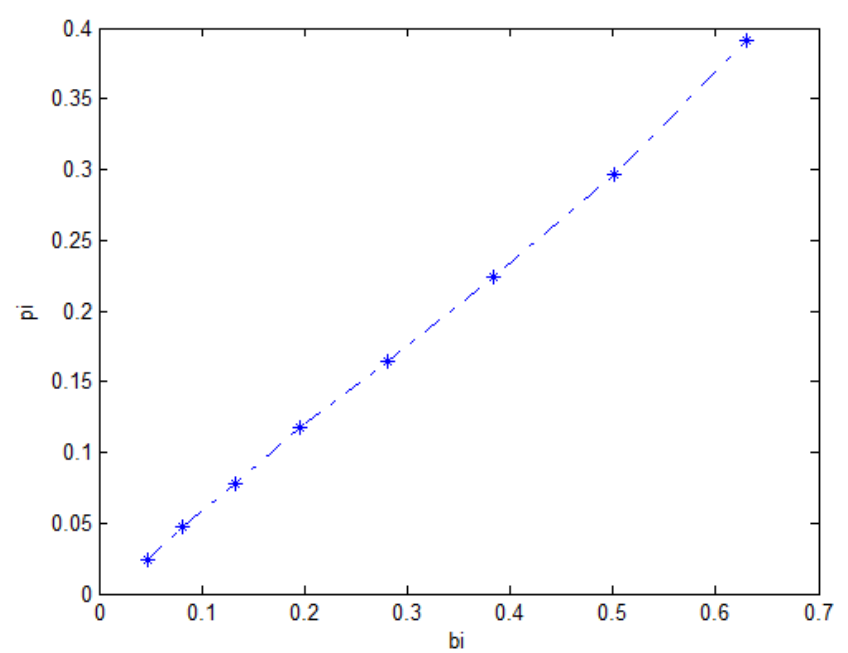

Fig. (3). The relationship between failure probability and hyperparameter.

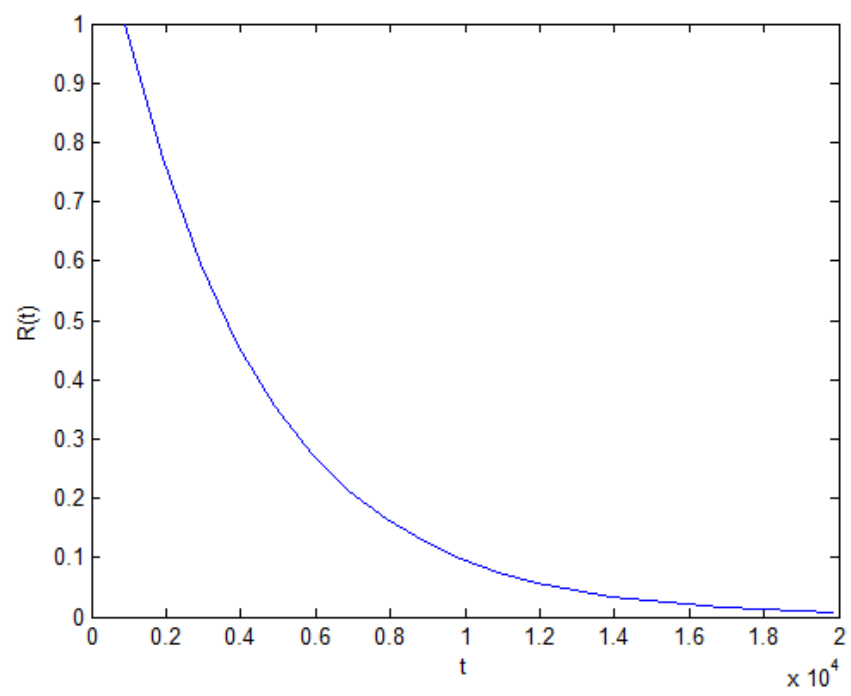

Fig. (4). The reliability curve of EMUs electromagnetic valve.

$\hat{\mu}=\frac{A D-B C}{D E-A B}=904.609$

$\hat{\sigma}=\frac{A^{2}-C E}{D E-A B}=3869.492$

$R(\mathrm{t})=\exp \left(-\frac{t-\hat{\mu}}{\hat{\sigma}}\right)=\exp \left(-\frac{t-904.609}{3869.492}\right), \quad t \geq 904.609$

$\lambda(\mathrm{t})=\frac{1}{\hat{\sigma}}=2.584 \times 10^{-4}$

Thus, the reliability curve of corresponding EMUs electromagnetic valve can be obtained as shown in Fig. (4).

\section{CONCLUSION}

This paper mainly researched on the parameter estimation of two-parameter exponential distribution, starting from the theoretical analysis, and concretely applying it to reliability evaluation of EMUs electromagnetic valve, as shown in Fig. (3), which can obtain estimation which is $R(1000)=0.9755$ with high reliability.

There is little difference between failure rate of an EMU which is $2.323 \times 10^{-4}$ and the estimated rate in this paper, which indicates that this method can basically make a precise estimate of failure rate. The application of this method can achieve the purpose of the reliability evaluation of electromagnetic valve, so there is certain theoretical significance for research on two-parameter exponential distribution for electromagnetic valve.

\section{CONFLICT OF INTEREST}

The author confirms that this article content has no conflict of interest.

\section{ACKNOWLEDGEMENTS}

This paper was supported by the national natural science fund project (51175028), Beijing outstanding talent training projects (2012D005017000006), the International Science \& Technology Cooperation Program of China (2014DFR70280) and BUCEA Urban Rural Construction and Management Industry Research Development Collabration Post Graduate Training Centre.

\section{REFERENCES}

[1] L. Zhu, and R.R. Liu. "The reliability evaluation of EMUs," Locomotive and Tolling Stock Technology, vol. 5, pp. 14-15, 2008.

[2] D.X. Zhu, and H.Z. Liu, "Reliability evaluation of life of high speed train bearing under mixed Beta distribution," Mechanical Science and Technology for Aerospace Engineering, vol. 33, no. 5, pp. 763-768, 2014.

[3] M. Han, "The construction and application of multilayer prior distribution function," Operations Research and Management Science, vol. 6, no. 3, pp. 31-40, 1997.

[4] M. Han, "The parameter estimation under zero-failure data for twoparameter exponential distribution," Operations Research and Management Science, vol. 7, no. 2, pp. 29-36, 1998.

[5] H.B. Zhao, "The statistical analysis of exponential distribution under zero-failure data," Applied Probability Statistics, vol. 20, no. 1, pp. 59-65, 2004.

[6] D.X. Zhu, and H. Liu, "Reliability evaluation of high-speed train bearing with minimum sample," Journal of Central South University Science and Technology, vol. 44, no. 3, pp. 963-969, 2013.

[7] E.Q. Hu, "Statistic inference of two-parameter exponential distribution under zero-failure data condition," Huazhong University of Science and Technology: Wuhan, 2005.

[8] Z.J. Dan, "The life and reliability evaluation of rolling bearing under zero-failure data condition," China Jiliang University: Hangzhou, 2013.

[9] N.G. Guo, X. Xu, and W.T. Jiao, "Zero-failure data analysis for exponential distribution," Journal of Zhengzhou University of Light Industry (Natural Science), vol. 23, no. 5, pp. 117-120, 2008.

[10] X.L. Wu, "The comparison of advantages and disadvantages parameters for two-parameter exponential distribution," Beijing Jiaotong University: Beijing, 2009.

[11] Q. Yin, H.B. Liu, and J.C. Liu, "The statistical analysis for twoparameter exponential distribution under zero-failure data," Journal of Huanggang Normal University, vol. 30, no. 3, pp. 37126, 2010.

[12] Z.H. Zhang, "The statistical analysis under zero-failure data condition," Mathematical Theory and Applied Probability, vol. 10, no. 1, pp. 94-101, 1995. 
[13] Z. Ji, "The statistical analysis under zero-failure data for exponential distribution," Acta Mathematicae Applicatae Sinica, vol. 31, no. 2, pp. 306-313, 2008.

[14] Z.H. Zhang, "Reliability theory and engineering application," Science Press: Beijing, 2012.
[15] M. Han, "Bayes estimation under zero-failure data for twoparemeter exponential distribution," Journal of mathematical research and exposition, vol. 19, no. 1, pp. 141-145, 1999.

Received: February 17, 2014

Revised: March 21, 2015

Accepted: June 9, 2015

(C) Yang et al.; Licensee Bentham Open.

This is an open access article licensed under the terms of the Creative Commons Attribution Non-Commercial License (http://creativecommons.org/licenses/by-nc/4.0/) which permits unrestricted, non-commercial use, distribution and reproduction in any medium, provided the work is properly cited. 